\title{
Bromodomain protein BRD4 is increased in human placentas from women with early-onset preeclampsia
}

\author{
Stella Liong ${ }^{1,2}$, Gillian Barker ${ }^{1,2}$ and Martha Lappas ${ }^{1,2}$ \\ 'Obstetrics, Nutrition and Endocrinology Group, Department of Obstetrics and Gynaecology, University of \\ Melbourne, Victoria, Australia and ${ }^{2}$ Mercy Perinatal Research Centre, Mercy Hospital for Women, Victoria, Australia
}

Correspondence should be addressed to M Lappas; Email: mlappas@unimelb.edu.au

\begin{abstract}
Preeclampsia affects $5 \%$ of all pregnancies and is a serious disorder of pregnancy, characterised by high maternal blood pressure, placental hypoxia, fluid retention (oedema) and proteinuria. Women with preeclampsia are associated with exaggerated levels of pro-inflammatory cytokines, chemokines and anti-angiogenic factors such as soluble fms-like tyrosine kinase-1 (sFLT1). Studies in non-gestational tissues have described the bromodomain (BRD) and extraterminal family of proteins, in particular BRD4 to play a critical role in propagating inflammation and is currently a therapeutic target for treating cancer, lung inflammation and asthma. The aims of this study were to: (i) determine the effect of severe early-onset preeclampsia on placental BRD4 expression; (ii) the effect of loss of BRD4 function by siRNA-targeted knockdown or with the BRD inhibitor JQ1 in human primary trophoblast cells and human umbilical vein endothelial cells (HUVECs) on TNF-stimulated production of pro-inflammatory mediators, cell adhesion molecules and anti-angiogenic markers and (iii) the effect of BRD4 suppression on placental sFLT1 secretion under hypoxia conditions and in preeclampic placenta. BRD4 mRNA expression was significantly increased (sevenfold) in severe early-onset preeclampsia placenta. BRD4 silencing resulted in a significant reduction in TNF-induced IL6, CXCL8, CCL2, CXCL1 and sFLT1-e15a mRNA expression and IL6, CXCL8, CCL2, CXCL1 and sFLT1 secretion in primary trophoblast and HUVECs. Additionally, JQ1 treatment significantly reduced placental sFLT1 secretion under hypoxic conditions and in preterm preeclamptic placenta. In conclusion, these findings suggest BRD4 may play a central role in propagating inflammation and endothelial dysfunction associated with the pathophysiology of earlyonset preeclampsia.

Reproduction (2018) 155 573-582
\end{abstract}

\section{Introduction}

Preeclampsia is a multisystem disorder affecting the maternal vasculature (causing hypertension), kidneys, liver and the feotoplacental unit (Roberts \& Hubel 2009). Preeclampsia is considered to be a two-stage disorder; abnormal placental implantation in early pregnancy is followed by maternal endothelial dysfunction (Young et al. 2010). For the mother, preeclampsia is a major contributor of maternal morbidity and mortality. For the baby, risk of preterm (PT) birth, intrauterine growth restriction and infant death are increased. There are no effective therapeutic treatments for preeclampsia. The only solution is the premature delivery of the foetus.

During early pregnancy, extravillous cytotrophoblasts invade the decidualised endometrium and myometrium and maternal spiral arteries to form large vessels of low resistance and is required for a successful pregnancy (Lyall et al. 2001). Placentae from women with severe early-onset preeclampsia have insufficient remodelling of maternal spiral arterioles. This failure in adequate spiral arteriole remodelling leads to chronic hypoxic conditions in the fetoplacental unit and increases in the secretion of anti-angiogenic factors into the maternal circulation. It is postulated that excessive inflammation and angiogenic imbalance play a role in the development of preeclampsia (Ramma \& Ahmed 2011). Studies have shown the anti-angiogenic factors such as soluble fms-like tyrosine kinase-1 (sFLT1) contribute to endothelial dysfunction, hypertension and proteinuria in preeclampsia (Maynard et al. 2003, Young et al. 2010). The association between inflammation and endothelial dysfunction has also been well documented. Studies have shown neutrophil activation is increased in preeclamptic patients (Prieto et al. 1997). In addition, circulating levels of pro-inflammatory cytokines such as IL6, TNF and the chemokines CXCL8 and CCL2 are also elevated in women with preeclampsia (Szarka et al. 2010).

The bromodomain and extraterminal (BET) family of proteins are described as critical mediators of transcription. Bromodomains (BRDs), which are present on BET proteins, are highly conserved functional domains that recognise and bind to acetylated lysine residues on histone tails. There are four members of the BRD family (BRD2, BRD3, BRD4 and BRDT), which 
recruit polymerases and transcription factors to activate gene transcription (Huang et al. 2009, Sanchez \& Zhou 2009). BRD4 is one of the well-studied member of the BET family. BRD4 recruits the positive transcription elongation factor $b$ and stimulates RNA polymerase II-dependent transcription (Zhang et al. 2012, Itzen et al. 2014). There is growing evidence that implicates BRDs to play a role in the development of various diseases by regulating the transcription of several genes involved in tumour growth and inflammation. Thus, there has been much interest on BRDs as therapeutic targets in cancer, lung inflammation and asthma (Khan et al. 2014, Wu et al. 2016, da Motta et al. 2017).

To date, there is only one study that has investigated the role of BRD4 in gestational tissues. This study identified inhibition of BRD4-suppressed vascular endothelial growth factor (VEGF-induced migration, proliferation and stress fibre formation in human umbilical vein endothelial cells (HUVECs; Huang et al. 2016). However, there are limited studies on the role of BRD4 in regulating placental inflammation and endothelial dysfunction associated with preeclampsia. Therefore, the aim of this study was to characterise placental BRD4 expression in early-onset preeclampsia and with hypoxia. The role of BRD4 on inflammation and the secretion of anti-angiogenic factors were assessed using a BRD inhibitor (JQ1) or BRD4-specific siRNA knockdown in primary trophoblast cells and HUVECs.

\section{Methods}

\section{Tissue collection}

Approval for this study was obtained from the Mercy Hospital for Women's Research and Ethics Committee, and written informed consent was obtained from all participating subjects. Women were invited to provide samples on the day of admission for surgery. For the expression studies, placenta was obtained from normotensive PT pregnancies $(n=18)$ and those complicated by severe early-onset preeclampsia $(n=19)$. Preeclamptics were diagnosed in accordance with the American College of Obstetricians and Gynecologists guidelines (The ACOG Task Force 2013). All samples were obtained from cases of early-onset preterm preeclampsia ( $<34$ weeks gestation). Women fulfilling any of the following criteria were excluded: vascular/renal complication, gestational diabetes mellitus, multiple gestations, asthma, smokers, chorioamnionitis, placental abruption, acute foetal distress and women with any other adverse underlying medical conditions. PT control placentas were selected from women with PT rupture of membranes, placenta praevia or antepartum haemorrhage and who did not have any evidence of infection (histopathologic examination of the placentas), hypertensive disease or maternal comorbidities. All patients delivered by caesarean section. Patient characteristics are outlined in Table 1.

Placenta was obtained and processed within $15 \mathrm{~min}$ of delivery. Placental lobules (cotyledons) were obtained from
Table 1 Patient characteristics.

\begin{tabular}{lcc}
\hline & PET $(n=19$ patients $)$ & $\begin{array}{c}\text { PT controls } \\
(n=18 \text { patients })\end{array}$ \\
\hline Maternal age & $30.7 \pm 1.1$ & $31.9 \pm 1.5$ \\
Pre-pregnancy BMI $\left(\mathrm{kg} / \mathrm{m}^{2}\right)$ & $25.1 \pm 2.1$ & $26.4 \pm 1.6$ \\
Delivery BMI $\left(\mathrm{kg} / \mathrm{m}^{2}\right)$ & $30.3 \pm 2.5$ & $29.0 \pm 1.5$ \\
Gestational age $($ weeks $)$ & $30.6 \pm 0.4^{*}$ & $33.0 \pm 0.6$ \\
Neonate birth weight $(\mathrm{g})$ & $1356 \pm 97^{*}$ & $2061 \pm 108$ \\
Fetal gender & $8: \mathrm{M} ; 11: \mathrm{F}$ & $9: \mathrm{M} ; 9: \mathrm{F}$ \\
Placental weight $(\mathrm{g})$ & $357 \pm 20^{*}$ & $459 \pm 37$ \\
Gravida & $1.4 \pm 0.2^{*}$ & $2.9 \pm 0.3$ \\
Parity & $1.3 \pm 0.1^{*}$ & $2.1 \pm 0.3$ \\
\hline
\end{tabular}

Values represent mean \pm S.E.M.

$\mathrm{M}$, male; F, female; ${ }^{*} P<0.05$ vs PT controls (Mann-Whitney $U$ test). PET, preterm preeclampsia; PT controls, normotensive preterm controls.

various locations of the placenta; the basal plate and chorionic surface were removed from the cotyledon; and villous tissue was obtained from the middle cross-section. Placental tissue was blunt dissected to remove visible connective tissue and calcium deposits. Tissues were washed extensively with PBS, and (i) immediately snap frozen in liquid nitrogen and stored at $-80^{\circ} \mathrm{C}$ for RNA analysis; (ii) fixed and paraffin embedded for immunohistochemical analysis or (iii) immediately used for explant studies or siRNA studies as detailed below.

\section{Placental explants}

Placental tissue explants were performed from normal healthy women at the time of term caesarean section ( $n=6$ patients). Placental tissue explants were performed immediately as previously described (Lim et al. 2013). Briefly, placenta was finely diced and transferred to 24-well tissue culture plates (20 mg/well). Tissues were incubated in the absence or presence of $5 \mu \mathrm{M}(+)-J Q 1$ (Cayman Chemical) for $60 \mathrm{~min}$ before the addition of $10 \mathrm{ng} / \mathrm{mL}$ TNF (PeproTech, Rocky Hill, NJ, USA) for $20 \mathrm{~h}$. For hypoxia explant studies, tissues were incubated in the absence or presence of $5 \mu \mathrm{M} J \mathrm{Q} 1$ in either hypoxic $\left(1 \% \mathrm{O}_{2}\right.$, $\left.5 \% \mathrm{CO}_{2}\right)$ or normoxic $\left(8 \% \mathrm{O}_{2}, 5 \% \mathrm{CO}_{2}\right)$ conditions for $20 \mathrm{~h}$ ( $n=6$ term normotensive patients; $n=4$ preterm preeclamptic (PET) patients). After final incubation, tissue and media were collected separately and stored at $-80^{\circ} \mathrm{C}$ for further analysis as detailed below. The concentration of JQ1 was based on previous studies (Shu et al. 2016).

\section{Isolation of primary villous trophoblast cells and umbilical vein endothelial cells}

Isolation and purification of primary villous trophoblast cells was performed using fresh placenta obtained from normal healthy pregnant women at term caesarean section. Placental villous cytotrophoblasts were isolated as previously described (Lappas 2014) by DNase/trypsin digestion and purified by separation on a Percoll gradient. Briefly, placental villous tissue $(\sim 25 \mathrm{~g})$ was dissected and washed in saline and then digested three times in a HEPES-buffered salt solution containing $0.25 \%$ trypsin and $0.2 \mathrm{mg} / \mathrm{mL}$ DNAse. Tissue was shaken at $37^{\circ} \mathrm{C}$ for $30 \mathrm{~min}$. The cytotrophoblast cells were separated on a Percoll 
gradient and resuspended in standard cell culture medium (5.5 mM glucose, 44.5\% DMEM, 44.5\% Ham's-F12, and 10\% foetal calf serum supplemented with antibiotics). The cells were plated on 24-well plates at a density of $5 \times 10^{5}$ cells per well. The cells were cultured for a total of $90 \mathrm{~h}$ at $37^{\circ} \mathrm{C}$ in $8 \%$ $\mathrm{O}_{2}$ and $5 \% \mathrm{CO}_{2}$ atmosphere, and the cell culture media was changed daily.

Isolation and purification of primary HUVECs was performed from umbilical cord from healthy pregnant women at term caesarean section. Isolation of HUVECs was performed as previously described (Crampton et al. 2007). Briefly, the umbilical vein was washed thoroughly with saline, and then incubated with $0.2 \%$ collagenase A for 10 min in a water bath. The detached endothelial cells were collected and resuspended in DMEM supplemented with $100 \mathrm{U} / \mathrm{mL}$ penicillin G, $100 \mu \mathrm{g} /$ $\mathrm{mL}$ streptomycin, $10 \%$ FBS (v/v), 1\% antibiotic-antimycotic $(\mathrm{v} / \mathrm{v}), 1 \%$ endothelial cell growth supplement $(\mathrm{v} / \mathrm{v})$ and $1 \%$ heparin (v/v). The cell suspension was incubated at $37^{\circ} \mathrm{C}$ in a humidified atmosphere of $5 \% \mathrm{CO}_{2}$ and $21 \% \mathrm{O}_{2}$.

\section{Gene silencing of Brd4 in primary trophoblast cells and HUVECs}

Transfection of trophoblast cells and HUVECs was performed as previously described (Lappas 2014) using BRD4 siRNA (siBRD4) and negative control siRNA (siCONT) obtained from Ambion (Thermo Fisher Scientific). Briefly, cells were transfected with $50 \mathrm{nM}$ siCONT or $50 \mathrm{nM}$ siBRD4 and $0.3 \%$ Lipofectamine 3000 (Life Technologies), incubated for $20 \mathrm{~h}$ and removed, and fresh medium was added to wells. After $66 \mathrm{~h}$ (total culture time), cells were treated with or without $10 \mathrm{ng} /$ $\mathrm{mLTNF}$, and the cells were incubated at $37^{\circ} \mathrm{C}$ for an additional $20 \mathrm{~h}$. Cells were collected and stored at $-80^{\circ} \mathrm{C}$ until assayed for mRNA expression by quantitative RT-PCR (qRT-PCR) as detailed below. Media was collected and stored at $-80^{\circ} \mathrm{C}$ until assayed for cytokine release as detailed below. MTT assay was performed on all treatments to determine any significant effects on cell viability. Experiments were performed on trophoblast cells and HUVECs isolated from five patients.

\section{Treatment of JQ1 in primary trophoblast cells and HUVECS}

Isolated trophoblast cells and HUVECs were also treated with $0.5 \mu \mathrm{M}$ JQ1 in the presence or absence of $10 \mathrm{ng} / \mathrm{mL} T N F$, following $66 \mathrm{~h}$ total culture time and cells were incubated at $37^{\circ} \mathrm{C}$ for an additional $20 \mathrm{~h}$. Cells were collected and stored at $-80^{\circ} \mathrm{C}$ until assayed for mRNA expression by qRT-PCR as detailed below. Media was collected and stored at $-80^{\circ} \mathrm{C}$ until assayed for cytokine release as detailed below. Experiments were performed on trophoblast cells and HUVECs isolated from five patients. For the hypoxia studies, isolated trophoblast cells following $66 \mathrm{~h}$ total culture time, were incubated with $0.5 \mu \mathrm{M}$ JQ1 in either hypoxic $\left(1 \% \mathrm{O}_{2}, 5 \% \mathrm{CO}_{2}\right)$ or normoxic $\left(8 \% \mathrm{O}_{2}, 5 \% \mathrm{CO}_{2}\right)$ conditions for $20 \mathrm{~h}$. After final incubation, cells and media were collected separately and stored at $-80^{\circ} \mathrm{C}$ for further analysis as detailed below. Cells and media were collected and stored at $-80^{\circ} \mathrm{C}$ until assayed for mRNA expression by qRT-PCR or assayed for cytokine release respectively, as detailed below. Hypoxia experiments were performed on trophoblast cells isolated from five patients.

\section{Immunohistochemistry}

To determine the expression of BRD4 in placenta, immunohistochemistry (IHC) was performed on paraffin sections as described previously using the IHC Select HRP Detection Set (Merck Millipore) (Lappas 2014). Briefly, sections were deparaffinised followed by an antigen retrieval step (boiled in $10 \mathrm{mM}$ Tris, $1 \mathrm{mM}$ EDTA, pH 9.0 for $10 \mathrm{~min}$ followed by 20 -min incubation) and then endogenous peroxidases were inactivated by adding 3\% hydrogen peroxide for $10 \mathrm{~min}$. After blocking (blocking reagent: normal goat serum in PBS) for $5 \mathrm{~min}$, sections were incubated with $0.2 \mu \mathrm{g} / \mathrm{mL}$ rabbit polyclonal anti-Brd4 (cat no. GTX130586; GeneTex, Irvine, CA, USA) in 1\% (wt/vol) bovine serum albumin in PBS and incubated in a humidity chamber at $4{ }^{\circ} \mathrm{C}$ overnight. Binding sites were labelled with biotin conjugated rabbit anti-goat IgG antibody followed by the streptavidin-HRP. Negative control slides, where primary antibody was replaced with rabbit IgG, were also performed.

\section{RNA extraction and $q R T-P C R$}

Total RNA was extracted from tissues using TRI Reagent according to manufacturer's instructions (Bioline, Alexandria, NSW, Australia), as previously described (Lappas 2014). RNA concentration and purity were measured using a NanoDrop ND1000 spectrophotometer (Thermo Scientific). RNA quality and integrity was determined using the $A_{260} / A_{280}$ ratio. RNA $(0.5 \mu \mathrm{g})$ was converted to cDNA using the high-capacity cDNA reverse transcription kit according to the manufacturer's instructions (Applied Biosystems). The RT-PCR was performed using the CFX384 Real-Time PCR detection system (Bio-Rad Laboratories) using $100 \mathrm{nM}$ of predesigned and validated QuantiTect BRD4, IL6, CXCL8, CXCL1 and CCL2 primers (primer sequences not available) (Qiagen). Quantification of sFLT1e15a and sFLT1-i13 genes were performed using previously published (Whitehead et al. 2011) primers: sFLT1-i13 primer sequence (forward 5'-ACAATCAGAGGTGAGCACTGCAA-3', reverse 5'-TCCGAGCCTGAAAGTTAGCAA-3') and sFlt-e15a primer sequence (forward 5'-CTCCTGCGAAACCTCAGTG-3', reverse 5'-GACGATGGTGACGTTGATGT-3'). Average gene $\mathrm{Ct}$ values were normalised against two housekeeping genes (succinate dehydrogenase complex flavoprotein subunit A (SDHA) and (tyrosine 3-monooxygenase/tryptophan 5-monooxygenase activation protein zeta (YWHAZ)). Fold differences were determined using the comparative Ct method.

\section{Enzyme immunoassays}

IL6 and CXCL8 cytokine release was assessed using CytoSet sandwich ELISA according to the manufacturer's instructions (Life Technologies). The release of sFlt, CXCL1, CCL2 and IL1B was performed using DuoSet sandwich ELISA according to the manufacturer's instructions (R\&D Systems). The inter- and intra-assay coefficients of variation for all assays were less than $10 \%$. 


\section{Statistical analysis}

Statistics was performed on the normalised data unless otherwise specified. All statistical analyses were undertaken using GraphPad Prism (GraphPad Software). For two sample comparisons, either a paired or unpaired Student's $t$ test was used to assess the statistical significance between normally distributed data; otherwise, the nonparametric Mann-Whitney $U$ (unpaired) or the Wilcoxon (matched-pairs) tests were used. For all other comparisons, the homogeneity of data was assessed by the Bartlett's test, and when significant, the data were logarithmically transformed before further analysis using a repeated-measures one-way ANOVA (with least significant difference post hoc testing to discriminate among the means). Statistical significance was ascribed to a $P$ value $<0.05$. Data were expressed as mean \pm S.E.M.

\section{Results}

\section{BRD4 expression is increased in preeclamptic placentas}

We first assessed the expression and cellular localisation of BRD4 in human placenta. Representative IHC images are shown in Fig. 1A. BRD4 was localised to the syncytiotrophoblast, stromal cells and endothelial cells from both PET and PT control placenta. No staining was present in the isotype $\lg G$ negative control. We next sought to characterise the expression of BRD4 in placenta from women with severe onlyonset preeclampsia. Human placenta was obtained from PT pregnancies not complicated by preeclampsia (PT control, $n=18$ ) and those complicated by severe early-onset preeclampsia (PET, $n=19$ ). Demographic characteristics for these populations are outlined in Table 1. As shown in Fig. 1B, placental BRD4 mRNA expression was significantly upregulated in the PET cohort compared to PT controls $(P=0.013)$. Placental hypoxia is a central feature of preeclampsia.(Young et al. 2010) Thus, we next determined whether hypoxia would alter BRD4 expression as BRD4 was mostly localised in the syncytiotrophoblast layer, trophoblast cells were isolated from healthy normotensive women and exposed to hypoxic conditions $\left(\begin{array}{lll}1 \% & \mathrm{O}_{2}\end{array}\right)$ overnight. Hypoxia significantly upregulated BRD4 mRNA expression in primary trophoblast cells by 1.9 -fold $(P=0.0203$; Fig. 1C). Several attempts to quantify BRD4 protein expression by western blotting with commercially available antibodies were unsuccessful.

\section{BRD4 inhibition decreases expression of pro-inflammatory cytokines in primary trophoblasts and in HUVECs}

Pregnancies complicated by preeclampsia are associated with exaggerated inflammation (Greer et al. 1994, Kupferminc et al. 1994, Harmon et al. 2016). To assess the effect of BRD4 on inflammation in placenta and HUVECs, we used two approaches: (i) BRD4-
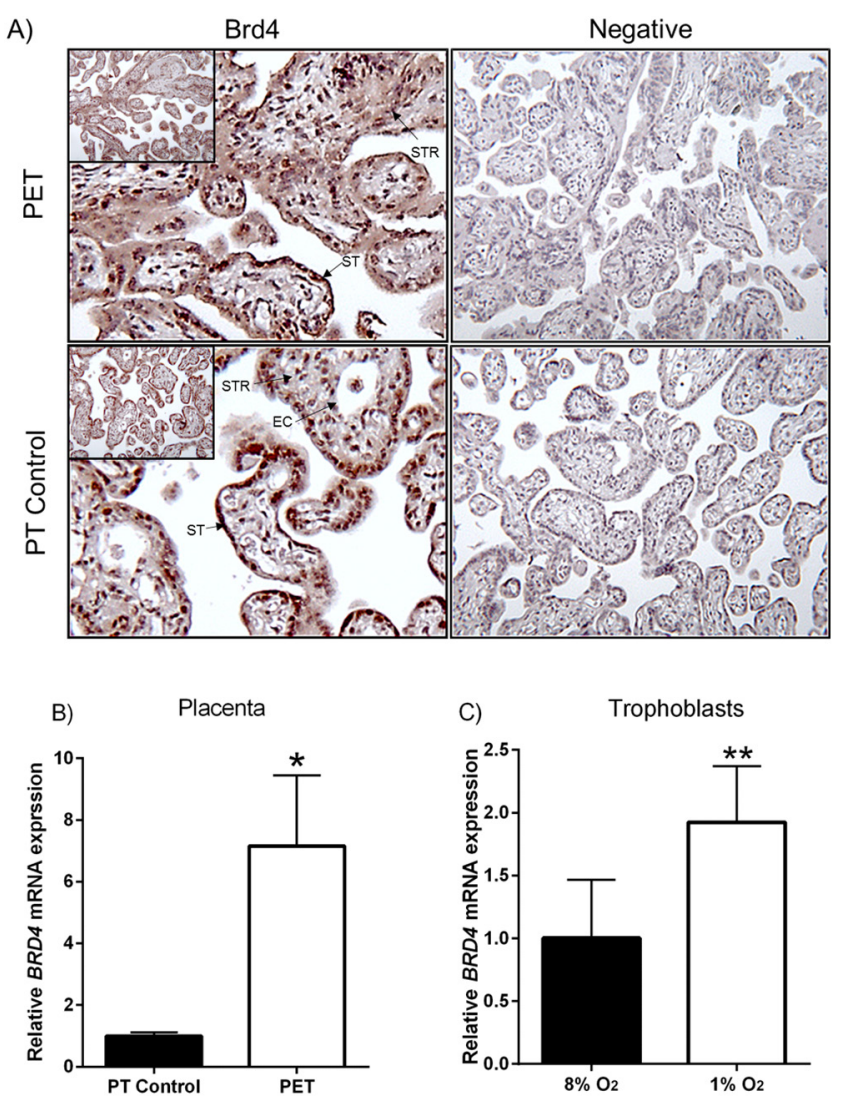

Figure 1 Localisation and expression of BRD4 in human preterm preeclamptic placenta. (A) Immunohistochemistry localisation of BRD4 was performed on human placenta obtained from preterm preeclamptic (PET; $n=6$ patients) and preterm normotensive control (PT control; $n=6$ patients) women at the time of term caesarean section. Representative IHC images of BRD4 immunoreactivity in placenta are shown. Arrows indicate immunoreactive BRD4 in syncytiotrophoblast (ST), endothelial cells lining the foetal capillaries (EC) and stromal cells (STR). BRD4: magnification 250x (insert 100x); Negative: magnification 100x. (B) BRD4 mRNA expression was assessed by qRT-PCR ( $n=18$ PT control; $n=19$ PET). Fold change was calculated relative to PT control. (C) Human primary trophoblast cells were incubated under normoxic $\left(8 \% \mathrm{O}_{2}\right)$ or hypoxic $\left(1 \% \mathrm{O}_{2}\right)$ conditions for $20 \mathrm{~h}$ ( $n=5$ patients). BRD4 mRNA expression was assessed by qRT-PCR. Fold change was calculated relative to $8 \% \mathrm{O}_{2}$ (normoxia). All data are displayed as mean \pm S.E.M. ${ }^{*} P<0.05$ vs PT control (Student's $t$ test); ${ }^{* *} P<0.05$ vs $8 \% \mathrm{O}_{2}$ (paired Student's $t$ test).

specific siRNA knockdown and (ii) treatment with the chemical inhibitor JQ1. First, we performed BRD4 siRNA knockdown in primary trophoblast cells and HUVECs and confirmed BRD4 silencing at the mRNA level in both primary trophoblast cells (66\% decrease) and HUVECs (48\% decrease). A MTT cell viability assay showed no difference in absorbance between cells transfected with siCONT or siBRD4 (data not shown).

The secretion of pro-inflammatory cytokines is very low under basal conditions. Therefore, trophoblast cells and HUVECs were treated with TNF. As shown in Fig. 2, TNF induced a robust and significant increase in mRNA expression and secretion of the pro-inflammatory 

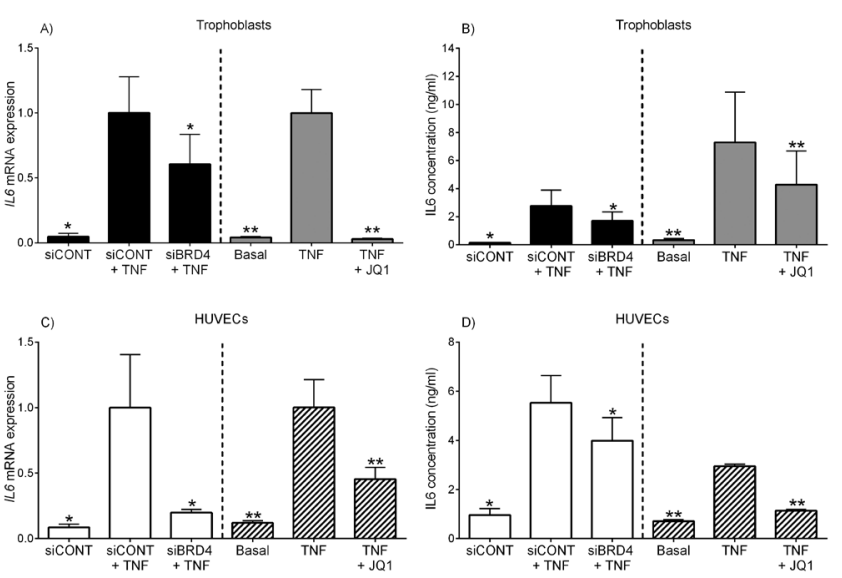

Figure 2 Effect of BRD4 silencing on TNF-induced pro-inflammatory cytokines in human primary trophoblast cells and HUVECs. (A and B) Human primary trophoblast cells and (C and D) HUVECs were either transfected with or without $100 \mathrm{nM}$ siBRD4 or siCONT for $48 \mathrm{~h}$ then treated with $10 \mathrm{ng} / \mathrm{mL}$ TNF for an additional $20 \mathrm{~h}$ ( $n=5$ patients) or incubated with $10 \mathrm{ng} / \mathrm{mL} T N F$ in the absence or presence of $0.5 \mu \mathrm{M}$ JQ1 for $20 \mathrm{~h}$ ( $n=5$ patients). (A and C) IL6 mRNA expression was analysed by qRT-PCR and the fold change was calculated relative to TNF. (B and D) The incubation medium was assayed for concentration of IL6, release by ELISA. All data are displayed as mean \pm S.E.M. ${ }^{*} P<0.05$ vs siCONT + TNF; ${ }^{* *} P<0.05$ vs TNF (one-way ANOVA).

cytokine IL6 from trophoblasts $(P<0.001)$ and HUVECs $(P<0.001$; Fig. 2). In trophoblast cells (Fig. 2A and B) and HUVECs (Fig. 2C and D) transfected with siBRD4, there was a significant decrease in TNF-induced mRNA expression $(P=0.019$ and $P=0.028$ respectively) and secretion ( $P<0.001$ and $P=0.031$ respectively) of IL6. In trophoblast cells (Fig. 2A and B) and HUVECs (Fig. 2C and D) treated with JQ1, TNF-induced IL6 mRNA expression ( $P=0.001$ and $P=0.011$ respectively) and secretion was also significantly reduced $(P=0.045$ and $P<0.001$ respectively).

\section{BRD4 inhibition decreases expression of chemokines in primary trophoblasts and in HUVECs}

We next assessed the effect of BRD4 silencing on the expression of chemokines in trophoblast and HUVECs. In trophoblast cells transfected with siBRD4, TNF induced mRNA expression and secretion of CXCL8 $(P=0.039$ and $P=0.040$ respectively; Fig. $3 \mathrm{~A}$ and $\mathrm{B})$, and CXCL1 $(P=0.026$ and $P<0.001$ respectively; Fig. 3E and $F$ ) was significantly reduced. BRD4 silencing in trophoblast cells had no significant effect on TNFinduced CCL2 mRNA expression and secretion (Fig. 3C and D). In contrast, JQ1 treatment of trophoblast cells also significantly reduced TNF-induced CCL2 mRNA expression $(P=0.032)$ and secretion $(P=0.007)$ in trophoblast cells (Fig. 3C and D). CXCL8 and CXCL1 secretion was also significantly reduced with JQ1 treatment in trophoblast cells $(P=0.003$ and $P=0.0032$
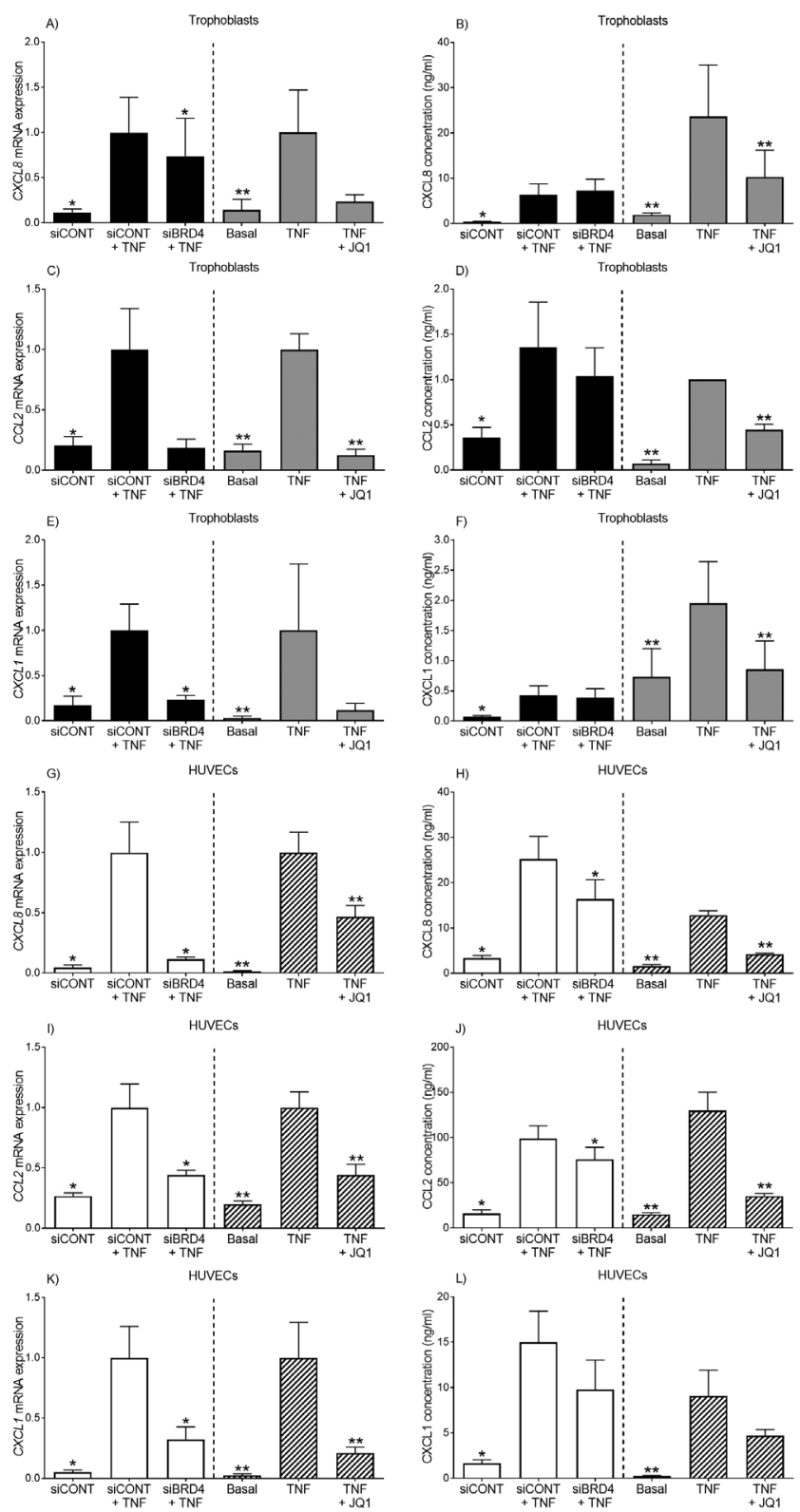

Figure 3 Effect of BRD4 silencing on TNF-induced pro-inflammatory chemokines in human primary trophoblast cells and HUVECs. (A, B, C, D, E and F) Human primary trophoblast cells and (G, H, I, J, K and L) HUVECs were either transfected with or without $100 \mathrm{nM}$ siBRD4 or siCONT for $48 \mathrm{~h}$ and then treated with $10 \mathrm{ng} / \mathrm{mL} \mathrm{TNF}$ for an additional $20 \mathrm{~h}$ ( $n=5$ patients) or incubated with $10 \mathrm{ng} / \mathrm{mL}$ TNF in the absence or presence of $0.5 \mu \mathrm{M}$ JQ1 for $20 \mathrm{~h}$ ( $n=5$ patients). (A, C, E, $\mathrm{G}, \mathrm{I}$ and $\mathrm{K})$ CXCL8, CXCL1 and CCL2 mRNA expression was analysed by $q R T-P C R$ and the fold change was calculated relative to TNF. (B,

$D, F, H, J$ and $L$ ) The incubation medium was assayed for concentration of CXCL8, CXCL1, CCL2 release by ELISA. All data are displayed as mean \pm S.E.M. ${ }^{*} P<0.05$ vs siCONT + TNF; ${ }^{*} P<0.05$ vs TNF (one-way ANOVA).

respectively; Fig. $3 \mathrm{~B}$ and $\mathrm{F}$ ); however, there was no change at the mRNA level (Fig. 3A and E).

In siCONT-transfected HUVECs, as expected, TNF significantly increased mRNA expression and 
secretion of CXCL8 $(P<0.001$ both; Fig. 3G and $\mathrm{H})$, CCL2 ( $P=0.004$ and $P=0.002$ respectively; Fig. 3I and $\mathrm{J})$ and $C X C L 1(P<0.001$ both; Fig. $3 \mathrm{~K}$ and $\mathrm{L})$. This increase in CCL2 by TNF mRNA expression $(P<0.001)$ and secretion $(P=0.046)$ was significantly reversed in siBRD4-transfected cells (Fig. 3G, H, I, J, K and L). CXCL8 mRNA expression was also significantly reduced in TNF-stimulated siBRD4-transfected cells $(P=0.018$, Fig. 3G). In contrast, there was no effect on TNF-induced CXCL8 secretion, nor with CXCL1 mRNA expression and secretion in siBRD4-transfected cells (Fig. 3L). On the other hand, TNF-induced CXCL8, CXCL1 and CCL2 mRNA expression $(P<0.001, P=0.009$ and $P=0.006$ respectively) and CXCL8 and CCL2 secretion $(P<0.001$ and $P=0.001$ respectively) was significantly reduced in HUVECs treated with JQ1 (Fig. 3G, H, I, J and K). In contrast, JQ1 treatment of HUVECs had no effect on CXCL1 secretion (Fig. 3L).

\section{BRD4 inhibition decreases sFLT1 in primary trophoblasts and in HUVECs}

Increased placental and endothelial release of antiangiogenic factors such as sFLT1 is associated with preeclampsia (Maynard et al. 2003, Young et al. 2010). Moreover, sFLT1 has been implicated to cause endothelial dysfunction and maternal organ injury (Maynard et al. 2003). Therefore, we next sought to assess the effect of loss of BRD4 on sFLT1 production in trophoblast cells and HUVECs. As has previously been reported (Xu et al. 2017), TNF does not increase either sFLT1 (e15a and $i 13$ variants) mRNA expression or sFLT1 secretion in trophoblast cells (Fig. 4). In siBRD4-transfected trophoblast cells, mRNA expression of the sFLT1-e15a variant and sFLT1 secretion were significant reduced $(P=0.045$ and $P=0.012$ respectively; Fig. $4 \mathrm{~B}$ and $\mathrm{C})$. There was no effect on the sFLT1-i13 variant with BRD4 knockdown (Fig. 4A). In trophoblast cells, sFLT1-e15a and sFLT1-i13 mRNA expression and sFLT1 secretion were also significantly reduced with JQ1 treatment $(P=0.027, P=0.019$ and $P=0.035$ respectively; Fig. 4A, $B$ and $C)$.

In addition to placenta, HUVECs are the second major source of circulating sFLT1. The effect of siBRD4 silencing was a significant reduction sFLT1-e15a mRNA expression ( $P=0.045$; Fig. 4E) and sFLT1 secretion $(P=0.036$; Fig. 4F). There was no effect of BRD4 silencing on the sFLT1-i13 variant (Fig. 4D). JQ1 treatment significantly reduced sFLT1-e15a $(P=0.008$; Fig. 4D) and sFLT1-i13 mRNA expression ( $P=0.002$; Fig. 4E) and sFLT1 secretion in HUVECs ( $P=0.013$; Fig. 4F).

\section{BRD4 inhibition decreases SFLT1 in response to hypoxia and in preeclamptic placenta}

Chronic placental hypoxia is also associated with preeclampsia (Makris et al. 2007). Together with
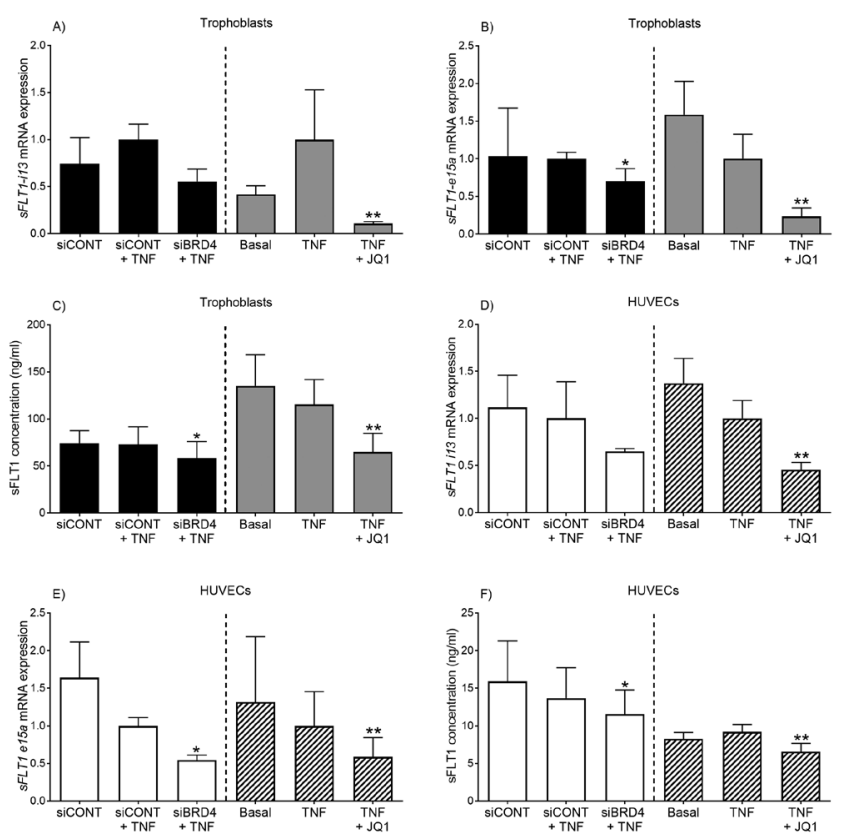

Figure 4 Effect of BRD4 silencing on TNF-induced sFLT1 production in human primary trophoblast cells and HUVECs. (A, B and C) Human primary trophoblast cells and (D, E and F) HUVECs were either transfected with or without $100 \mathrm{nM}$ siBRD4 or siCONT for $48 \mathrm{~h}$ and then treated with $10 \mathrm{ng} / \mathrm{mL}$ TNF for an additional $20 \mathrm{~h}(n=5$ patients) or incubated with $10 \mathrm{ng} / \mathrm{mL}$ TNF in the absence or presence of $0.5 \mu \mathrm{M}$ JQ1 for $20 \mathrm{~h}$ ( $n=5$ patients). (A, B, D and E) sFLT1-i13 and sFLT1-e15a mRNA expression was analysed by qRT-PCR and the fold change was calculated relative to TNF. ( $C$ and F) The incubation medium was assayed for concentration of sFLT1 release by ELISA. All data are displayed as mean \pm S.E.M. ${ }^{*} P<0.05$ vs siCONT +TNF; $* * P<0.05$ vs TNF (one-way ANOVA).

our observation that BRD4 expression is increased with hypoxia and in PET placenta, we next sought to investigate whether JQ1 may alleviate sFLT1 production associated with hypoxia. First, primary trophoblast cells were treated with JQ1 and exposed to hypoxic $\left(1 \% \mathrm{O}_{2}\right)$ conditions overnight. As expected, trophoblast cells incubated under hypoxic conditions had significantly elevated sFLT1-e15a $(P=0.008$; Fig. 5A $)$ and SFLT1-i13 mRNA expression ( $P=0.040$; Fig. 5B) compared to cells incubated under normoxic $\left(8 \% \mathrm{O}_{2}\right)$ conditions. In contrast, there was no increase in SFLT1 secretion by trophoblast cells exposed to $1 \% \mathrm{O}_{2}$ overnight (Fig. 5C). JQ1 treatment however significantly reduced $S F L T 1-e 15$ a $(P=0.033$; Fig. 5A) and sFLT1-i13 mRNA expression $(P=0.012$; Fig. 5B) and sFLT1 secretion $(P=0.034$; Fig. 5C) in trophoblast cells exposed to hypoxia.

In addition to trophoblast cells, placental explants were also performed on term healthy women or PET women. Tissues were treated with JQ1 and incubated in either 1\% (hypoxia) or 8\% (normoxia) oxygen overnight. As shown in Fig. 5D, in healthy normotensive term placenta, sFLT1 secretion was significantly elevated under hypoxic conditions $(P=0.002)$. This increase 

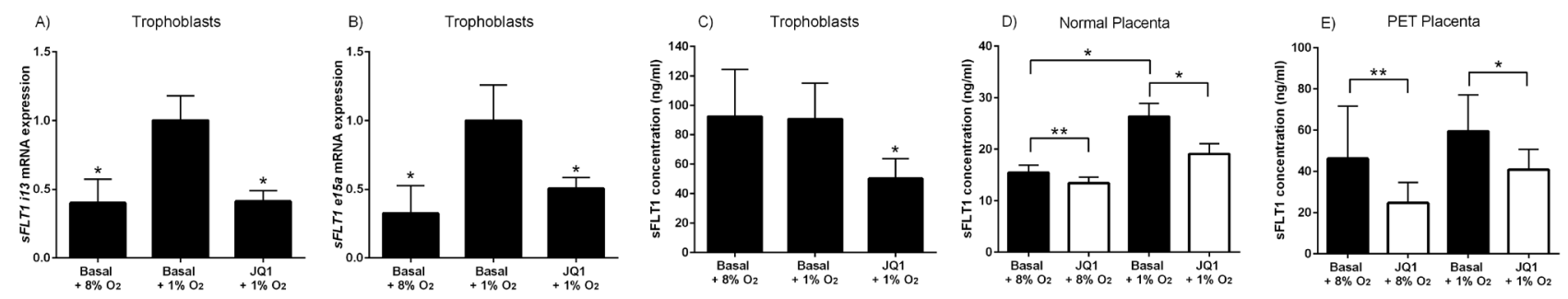

Figure 5 Effect of BRD4 inhibitor JQ1 on hypoxia-induced sFLT1 production in human primary trophoblasts and in preterm preeclamptic placenta. (A, B and C) Human primary trophoblast cells were incubated under normoxic $\left(8 \% \mathrm{O}_{2}\right)$ or hypoxic $\left(1 \% \mathrm{O}_{2}\right)$ conditions for $20 \mathrm{~h}(n=5$ patients). sFLT1-i13 and sFLT1-e15a mRNA expression was assessed by qRT-PCR. Fold change was calculated relative to $1 \% \mathrm{O}_{2}$ (hypoxia). The incubation medium was assayed for concentration of sFLT1 release by ELISA. (D and E) Human placentae were obtained from (D) healthy term women (normal) or from (E) women with early-onset preeclampsia (PET). Placental tissues were incubated under normoxic $\left(8 \% \mathrm{O}_{2}\right)$ or hypoxic $\left(1 \% \mathrm{O}_{2}\right)$ conditions in the absence or presence of $5 \mu \mathrm{M} \mathrm{JQ} 1$ for $20 \mathrm{~h}(n=5$ patients). The incubation medium was assayed for concentration of SFLT1 release by ELISA. All data are displayed as mean \pm S.E.M. ${ }^{*} P<0.05$ vs basal $+1 \% \mathrm{O}_{2} ;{ }^{* *} P<0.05$ vs basal $+8 \% \mathrm{O}_{2}$ (one-way ANOVA).

in sFLT1 under hypoxia was significantly reversed following JQ1 treatment $(P<0.001)$. Moreover, sFLT1 secretion was also significantly decreased under normoxic conditions with JQ1 treatment $(P<0.001)$. In comparison to normotensive term placenta, sFLT1 secretion did not increase following incubation under hypoxic conditions in PET placenta (Fig. 5E). However, sFLT1 secretion was significantly reduced with JQ1 treatment of PET placenta under hypoxic $(P=0.023)$ and normoxic $(P=0.035)$ conditions (Fig. 5E).

\section{Discussion}

Preeclampsia is a serious complication of pregnancy that is associated with placental hypoxia, and increased inflammation and secretion of anti-angiogenic factors. To our knowledge, this is the first study to examine the role of the BET bromodomain protein BRD4 in preeclampsia. In this study, we report placental BRD4 expression to be significantly elevated with preeclampsia. We also report that placental BRD4 expression is upregulated by hypoxia and acts as an upstream regulator of trophoblast and HUVEC production of pro-inflammatory cytokines, chemokines and the anti-angiogenic factor sFLT1. Furthermore, treatment of preeclamptic placenta with BRD4 inhibitor JQ1 resulted in a significant decrease in sFLT1 production under normoxic and hypoxic conditions. Thus, our findings suggest that placental BRD4 may contribute to the pathophysiology of preeclampsia by acting as an upstream regulator of inflammation and anti-angiogenetic factors that are associated with this disease.

Preeclampsia is associated with poor trophoblast invasion and inadequate spiral artery remodelling, which leads to the development of placental hypoxia (Roberts \& Hubel 2009, Young et al. 2010). It is hypothesised that placental hypoxia plays a crucial role in initiating the cascade of events that results in the clinical manifestations of the disease (Young et al. 2010). In this study, we report BRD4 expression is significantly increased in preeclamptic placenta (7-fold increase) as well as in human primary trophoblast cells when exposed to hypoxia (1.9-fold increase). Alterations in BRD4 expression under hypoxic conditions has been previously reported in non-gestational tissues (da Motta et al. 2017). In agreement to our findings, da Motta et al. (2017) reported that BRD4 expression is upregulated with hypoxia and that JQ1 treatment profoundly supressed hypoxia-responsive gene expression in breast cancer cells. These hypoxic JQ1-regulated genes were identified to be bound by BRD4 and are direct targets of either HIF1A or HIF2A. Taken together, our study and the study by da Motta et al. (2017) demonstrate BRD4 expression is increased with hypoxia and in turn may upregulate the expression of hypoxia-responsive genes.

Studies have shown hypoxic primary trophoblast cells secrete elevated levels of sFLT1 (Li et al. 2005). It is hypothesised that excessive secretion of anti-angiogenic factors such as sFlt1 in preeclamptic placenta is an attempt to improve blood flow to the fetoplacental unit (Maynard \& Karumanchi 2011); however, the upstream mechanisms in regulating sFLT1 secretion associated with preeclampsia remains unclear. In this study, we found that BRD4 silencing resulted in a significant reduction in SFLT1 production in primary trophoblast cells. A recent study by Shackleford et al. (2017) also identified BRD4 to induce FLT1 mRNA expression in paediatric sarcoma cell lines. Taken together, our findings suggest blockade of BRD4 with JQ1 could alleviate the excessive production of sFLT1 in trophoblast cells in response to hypoxia and in preeclampsia. In this study, hypoxia significantly elevated SFLT1-e15a and SFLT1-i13 mRNA expression in trophoblast cells, which was subsequently reversed with JQ1 treatment. However, we did not find sFLT1 secretion by trophoblasts to be increased with hypoxia. This is in contrast to studies by Li et al. (2005) and Kaitu'u-Lino et al. (2014), in which they demonstrated increased sFLT1 secretion in trophoblast cells cultured under hypoxia. This difference in hypoxia-induced sFLT1 secretion in trophoblast cells could be reflective of differences in experimental design. Notwithstanding this limitation, we found JQ1 treatment 
significantly decreased sFLT1 secretion in trophoblast cells exposed to hypoxia.

In addition to trophoblast cells, we assessed the effect of JQ1 on hypoxia-induced sFLT1 production in placentas obtained from normal and preeclamptic women. Intriguingly, compared to normotensive women, placentas from preeclamptic women did not show elevated sFLT1 secretion in response to $1 \%$ (hypoxia) vs 8\% (normoxia) oxygen conditions. Given that preeclamptic placentas already secrete high levels of sFLT1 compared to normal healthy placentas, subsequent insults from incubating tissues under hypoxic conditions may not further augment sFLT1 secretion. Nevertheless, we found elevated sFLT1 production in placental explants exposed to $1 \%$ oxygen tensions were subsequently suppressed with JQ1 treatment. Thus, we propose that increased BRD4 expression induced by hypoxic conditions may upregulate sFLT1 production in preeclampsia, and in turn upregulate the expression of hypoxia-responsive genes including sFLT1. Further studies will also be needed to determine whether the effects of BRD4-induced sFLT1 production in sFLT1 in trophoblast and HUVECs are mediated via VEGFR1.

Endothelial dysfunction is another key feature of preeclampsia (Brodowski et al. 2017). Excluding the placenta, endothelial cells are the next major source of sFLT1 production. sFLT1 is a negative regulator of endothelial function (Ahmad et al. 2011). For example, sFLT1 blockade restored both basal and VEGF-Amediated migration and tube formation in HUVECs (Ahmad et al. 2011). In this study, BRD4 silencing in HUVECs significantly decreased sFLT1 in HUVECs. To our knowledge, there is only one other study to assess the role of BRD4 in HUVECs. In this study, Huang et al. (2016) found JQ1 treatment suppressed angiogenesis in HUVECs. This is in contract to our study where we found BRD4 silencing in HUVECs suppressed the production of the anti-angiogenic factor sFLT1. Further studies are warranted to elucidate the role of BRD4 in HUVECs of preeclamptic women.

Preeclampsia is also associated with chronic inflammation. Women with preeclampsia have higher circulating levels of TNF and IL6 compared to healthy women (Greer et al. 1994, Kupferminc et al. 1994). It is hypothesised that during preeclampsia, chronic placental hypoxia occurs as a consequence of shallow trophoblast invasion and an immune imbalance of where $\mathrm{T}$ regulatory cells are decreased and pro-inflammatory CD4 ${ }^{+}$T-cells are increased (Harmon et al. 2016). This immune imbalance leads to increased oxidative stress and pro-inflammatory cytokines resulting in chronic inflammation. Moreover, there is evidence that suggests sFLT1 and TNF secreted by the placenta, in response to oxidative stress, sensitises HUVECs to proinflammatory mediators which subsequently leads to maternal endothelial cell dysfunction (Cindrova-Davies et al. 2011). Previous studies have demonstrated BRD4 to mediate inflammation (Khan et al. 2014, Tian et al. 2017). To our knowledge, there have been no studies on the role of BRD4 on inflammation in trophoblast cells or HUVECs. Thus, we next sought to investigate the role of BRD4 in mediating inflammation associated with preeclampsia. Loss of BRD4 resulted in reduced TNFinduced expression of IL6, CXCL8, CXCL1 and CCL2 in primary trophoblast cells and HUVECs. Thus, given the importance of inflammation in the pathophysiology of preeclampsia, we propose that BRD4 may act as an upstream regulator of inflammation associated with preeclampsia. Further studies are required to confirm if BRD4 regulation of inflammation and sFLT1 is unique to preeclampsia or due to activation of the hypoxia/VEFG signalling cascade.

A limitation of this study was that we were unable to quantify BRD4 protein expression in human placenta. Several attempts to quantify BRD4 protein expression with commercially available antibodies were unsuccessful. A limitation of this study is that we are unable to determine the temporal expression of placental BRD4 across different stages of pregnancy in normal and PET pregnancies. This will be important to elucidate whether dysregulation of BRD4 expression occurs during early or late stages of pregnancy. In addition, these studies should also assess the cell types that express BRD4 throughout pregnancy. In situ hybridisation studies in placental sections are also required to confirm BRD4 expression in the different cell types in human placenta. Notwithstanding these limitations, a strength of our study is our wellcharacterised cohort of placental samples. All the early-onset preeclampsia and PT control samples were obtained from women at caesarean section (in the absence of labour), and patients were excluded for clinical factors which may influence BRD4 expression such as obesity, asthma, infection and diabetes.

The novel findings from this study demonstrate that increased BRD4 expression may play a role in the pathogenesis of preeclampsia. Notably, there are a number of clinical trials investigating BRD inhibitors as treatments for various cancers. There are currently 16 active clinical trials that are in various phases of development against haematological and solid tumours. Further investigation, however, using in vivo animal models are required to investigate whether JQ1 or other BRD inhibitors may be of therapeutic benefit preventing the pathogenesis of early-onset preeclampsia.

\section{Declaration of interest}

The authors declare that there is no conflict of interest that could be perceived as prejudicing the impartiality of the research reported. 


\section{Funding}

Associate Prof. Martha Lappas is supported by Fellowships from the National Health and Medical Research Council (Career Development Fellowship grant no. 1047025) and University of Melbourne (MDHS Research Fellowship). Funding for this study was provided by the Norman Beischer Medical Research Foundation, the University of Melbourne and the Mercy Research Foundation.

\section{Acknowledgements}

The following are gratefully acknowledged: the clinical Research Midwives Genevieve Christophers, Gabrielle Pell and Rachel Murdoch for sample collection; the Obstetrics and Midwifery staff of the Mercy Hospital for Women for their cooperation.

\section{References}

Ahmad S, Hewett PW, Al-Ani B, Sissaoui S, Fujisawa T, Cudmore MJ \& Ahmed A 2011 Autocrine activity of soluble Flt-1 controls endothelial cell function and angiogenesis. Vascular Cell 3 15. (https://doi. org/10.1186/2045-824X-3-15)

Brodowski L, Burlakov J, Hass S, von Kaisenberg C \& von VersenHoynck F 2017 Impaired functional capacity of fetal endothelial cells in preeclampsia. PLoS ONE 12 e0178340. (https://doi.org/10.1371/journal. pone.0178340)

Cindrova-Davies T, Sanders DA, Burton GJ \& Charnock-Jones DS 2011 Soluble FLT1 sensitizes endothelial cells to inflammatory cytokines by antagonizing VEGF receptor-mediated signalling. Cardiovascular Research 89 671-679. (https://doi.org/10.1093/cvr/cvq346)

Crampton SP, Davis J \& Hughes CC 2007 Isolation of human umbilical vein endothelial cells (HUVEC). Journal of Visualized Experiments 3183. (https://doi.org/10.3791/183)

da Motta LL, Ledaki I, Purshouse K, Haider S, De Bastiani MA, Baban D, Morotti M, Steers G, Wigfield S, Bridges E et al. 2017 The BET inhibitor JQ1 selectively impairs tumour response to hypoxia and downregulates CA9 and angiogenesis in triple negative breast cancer. Oncogene 36 122-132. (https://doi.org/10.1038/onc.2016.184)

Greer IA, Lyall F, Perera T, Boswell F \& Macara LM 1994 Increased concentrations of cytokines interleukin-6 and interleukin-1 receptor antagonist in plasma of women with preeclampsia: a mechanism for endothelial dysfunction? Obstetrics and Gynecology 84 937-940.

Harmon AC, Cornelius DC, Amaral LM, Faulkner JL, Cunningham MW Jr, Wallace K \& LaMarca B 2016 The role of inflammation in the pathology of preeclampsia. Clinical Science 130 409-419. (https://doi.org/10.1042/ CS20150702)

Huang B, Yang XD, Zhou MM, Ozato K \& Chen LF 2009 Brd4 coactivates transcriptional activation of NF-kappaB via specific binding to acetylated RelA. Molecular and Cellular Biology 29 1375-1387. (https://doi. org/10.1128/MCB.01365-08)

Huang M, Qiu Q, Xiao Y, Zeng S, Zhan M, Shi M, Zou Y, Ye Y, Liang L, Yang X et al. 2016 BET bromodomain suppression inhibits VEGF-induced angiogenesis and vascular permeability by blocking VEGFR2-mediated activation of PAK1 and eNOS. Scientific Reports 6 23770. (https://doi. org/10.1038/srep23770)

Itzen F, Greifenberg AK, Bosken CA \& Geyer M 2014 Brd4 activates P-TEFb for RNA polymerase II CTD phosphorylation. Nucleic Acids Research 42 7577-7590. (https://doi.org/10.1093/nar/gku449)

Kaitu'u-Lino TJ, Tong S, Beard S, Hastie R, Tuohey L, Brownfoot F, Onda K \& Hannan NJ 2014 Characterization of protocols for primary trophoblast purification, optimized for functional investigation of sFlt-1 and soluble endoglin. Pregnancy Hypertension 4 287-295. (https://doi. org/10.1016/j.preghy.2014.09.003)

Khan YM, Kirkham P, Barnes PJ \& Adcock IM 2014 Brd4 is essential for IL1beta-induced inflammation in human airway epithelial cells. PLOS ONE 9 e95051. (https://doi.org/10.1371/journal.pone.0095051)
Kupferminc MJ, Peaceman AM, Wigton TR, Rehnberg KA \& Socol ML 1994 Tumor necrosis factor-alpha is elevated in plasma and amniotic fluid of patients with severe preeclampsia. American Journal of Obstetrics and Gynecology 170 1752-1757. (https://doi.org/10.1016/ S0002-9378(12)91845-1)

Lappas M 2014 The NR4A receptors Nurr1 and Nur77 are increased in human placenta from women with gestational diabetes. Placenta 35 866-875. (https://doi.org/10.1016/j.placenta.2014.08.089)

Li H, Gu B, Zhang Y, Lewis DF \& Wang Y 2005 Hypoxia-induced increase in soluble Flt-1 production correlates with enhanced oxidative stress in trophoblast cells from the human placenta. Placenta 26 210-217. (https://doi.org/10.1016/j.placenta.2004.05.004)

Lim R, Barker G, Wall CA \& Lappas M 2013 Dietary phytophenols curcumin, naringenin and apigenin reduce infection-induced inflammatory and contractile pathways in human placenta, foetal membranes and myometrium. Molecular Human Reproduction 19 451-462. (https://doi. org/10.1093/molehr/gat015)

Lyall F, Bulmer JN, Duffie E, Cousins F, Theriault A \& Robson SC 2001 Human trophoblast invasion and spiral artery transformation: the role of PECAM-1 in normal pregnancy, preeclampsia, and fetal growth restriction. American Journal of Pathology 158 1713-1721. (https://doi. org/10.1016/S0002-9440(10)64127-2)

Makris A, Thornton C, Thompson J, Thomson S, Martin R, Ogle R, Waugh R, McKenzie P, Kirwan P \& Hennessy A 2007 Uteroplacental ischemia results in proteinuric hypertension and elevated sFLT-1. Kidney International 71 977-984. (https://doi.org/10.1038/sj.ki.5002175)

Maynard SE \& Karumanchi SA 2011 Angiogenic factors and preeclampsia. Seminars in Nephrology 31 33-46. (https://doi.org/10.1016/j. semnephrol.2010.10.004)

Maynard SE, Min JY, Merchan J, Lim KH, Li J, Mondal S, Libermann TA, Morgan JP, Sellke FW, Stillman IE et al. 2003 Excess placental soluble fms-like tyrosine kinase 1 (sFlt1) may contribute to endothelial dysfunction, hypertension, and proteinuria in preeclampsia. Journal of Clinical Investigation $\mathbf{1 1 1}$ 649-658. (https://doi.org/10.1172/ JCl17189)

Prieto JA, Panyutich AV \& Heine RP 1997 Neutrophil activation in preeclampsia. Are defensins and lactoferrin elevated in preeclamptic patients? Journal of Reproductive Medicine 42 29-32.

Ramma W \& Ahmed A 2011 Is inflammation the cause of pre-eclampsia? Biochemical Society Transaction 39 1619-1627. (https://doi. org/10.1042/BST20110672)

Roberts JM \& Hubel CA 2009 The two stage model of preeclampsia: variations on the theme. Placenta 30 (Supplement A) S32-S37. (https:// doi.org/10.1016/j.placenta.2008.11.009)

Sanchez R \& Zhou MM 2009 The role of human bromodomains in chromatin biology and gene transcription. Current Opinion in Drug Discovery and Development 12 659-665.

Shackleford TJ, Hariharan S, Phelps D, Bid H, Zhong H \& Houghton P 2017 Abstract 5825: mechanisms of intrinsic and acquired resistance to antibodies targeting IGF-1R in pediatric sarcoma cell lines. Cancer Research 77 5825. (https://doi.org/10.1158/1538-7445. AM2017-5825)

Shu S, Lin CY, He HH, Witwicki RM, Tabassum DP, Roberts JM, Janiszewska M, Huh SJ, Liang Y, Ryan J et al. 2016 Response and resistance to BET bromodomain inhibitors in triple-negative breast cancer. Nature 529 413-417. (https://doi.org/10.1038/nature16508)

Szarka A, Rigo J Jr, Lazar L, Beko G \& Molvarec A 2010 Circulating cytokines, chemokines and adhesion molecules in normal pregnancy and preeclampsia determined by multiplex suspension array. BMC Immunology 11 59. (https://doi.org/10.1186/1471-2172-11-59)

The ACOG Task Force, posting date 2013 American College of Obstetricians and Gynecologists guidelines. (available at: https://www.acog.org/ Resources-And-Publications/Task-Force-and-Work-Group-Reports/ Hypertension-in-Pregnancy) (Online.)

Tian B, Yang J, Zhao Y, Ivanciuc T, Sun H, Garofalo RP \& Brasier AR 2017 BRD4 couples NF-kappaB/RelA with airway inflammation and the IRF-RIG-I amplification loop in respiratory syncytial virus infection. Journal of Virology 91 e00007-e00017. (https://doi.org/10.1128/ JVI.00007-17)

Whitehead CL, Palmer KR, Nilsson U, Gao Y, Saglam B, Lappas M \& Tong S 2011 Placental expression of a novel primate-specific splice variant of sFlt-1 is upregulated in pregnancies complicated by severe early onset 
pre-eclampsia. BJOG 118 1268-1271. (https://doi.org/10.1111/j.14710528.2011.02962.x)

Wu X, Liu D, Tao D, Xiang W, Xiao X, Wang M, Wang L, Luo G, Li Y, Zeng $\mathbf{F}$ et al. 2016 BRD4 regulates EZH2 transcription through upregulation of C-MYC and represents a novel therapeutic target in bladder cancer. Molecular Cancer Therapeutics 15 1029-1042. (https:// doi.org/10.1158/1535-7163.MCT-15-0750)

Xu B, Shanmugalingam R, Chau K, Pears S, Hennessy A \& Makris A 2017 The effect of acetyl salicylic acid (Aspirin) on trophoblast-endothelial interaction in vitro. Journal of Reproductive Immunology 124 54-61. (https://doi.org/10.1016/j.jri.2017.10.044)

Young BC, Levine RJ \& Karumanchi SA 2010 Pathogenesis of preeclampsia. Annual Review of Pathology 5 173-192. (https://doi.org/10.1146/ annurev-pathol-121808-102149)
Zhang W, Prakash C, Sum C, Gong Y, Li Y, Kwok JJ, Thiessen N, Pettersson S, Jones SJ, Knapp S et al. 2012 Bromodomain-containing protein 4 (BRD4) regulates RNA polymerase II serine 2 phosphorylation in human CD4+ T cells. Journal of Biological Chemistry 287 43137-43155. (https://doi. org/10.1074/jbc.M112.413047)

Received 7 December 2017

First decision 1 January 2018

Revised manuscript received 26 March 2018

Accepted 16 April 2018 